\title{
A THEORETICAL INVESTIGATION OF THE Si - Si DOUBLE BOND IN A SERIES OF SUBSTITUTED DISILENES
}

\author{
L. Andersen, M. Dabney, and H.A. Harris* \\ Department of Chemistry, Creighton University, Omaha, NE 68178-0104, USA
}

\begin{abstract}
The nature of the Si-Si double bond in a number of substituted disilenes $\left(\mathrm{R}_{2} \mathrm{Si}=\mathrm{SiR}\right.$, $\mathrm{R}=\mathrm{H}, \mathrm{CH}_{3}$, $t$-butyl, mesityl) was investigated using the Fenske-Hall molecular orbital method. The results of the electronic structure calculations indicate that alkyl substituents serve to stabilize the Si-Si double bond electronically as well as sterically, while aryl substituents appear to destabilize the Si-Si bond. Calculations including d-orbital functions in the Si basis set show that the availability of $d$-orbitals on Si plays an important role in altering the compositions and lowering the energies of the virtual molecular orbitals. This result is in agreement with the experimentally observed spectroscopic transitions in the visible region and may also account for the relatively low rotational barriers that have been observed in the experimentally determined disilenes.
\end{abstract}

\section{Introduction}

Compounds containing multiple bonds between second-row elements $(P, S i)$ are of considerable current interest ${ }^{1}$ to both experimental and theoretical chemists. Molecules of this type have been proposed since 1877 although they have only recently been isolated and characterized. The first of these compounds to be unambiguously characterized was tetramesityldisilene, reported by West and coworkers in $1981 .{ }^{2}$ Since then a number of disilenes, all stabilized by bulky substituents, have been synthesized and structurally characterized. $3-10$ While the disilenes are more reactive than their alkene counterparts, the general reactivity trends are similar. Disilenes structures can differ markedly from the structures of analogous alkenes. The Si-Si bond distances in disilenes show the same degrees of shortening (relative to the known Si-Si single bond lengths in silanes) as do C-C distances in alkenes relative to alkanes but disilenes are, in general, non planar, exhibiting both twisting and bending about the Si-Si axis. ${ }^{11}$

Disilenes provide an interesting and challenging problem for the theoritician. The Si-Si bond lengths (determined by single-crystal X-ray studies) clearly indicate some degree of multiple bonding; however, the nonplanarity of disilenes and the low barrier to rotation about the $\mathrm{Si}-\mathrm{Si}$ axis call into question the usual double bond assignment of one $\sigma$ - and one $\pi$ - bond. Furthermore the "Classical Double Bond Rule," as stated in many inorganic chemistry textbooks, implies that $\pi$ bonding using Si d-orbitals may be an important factor in the bonding of these compounds. ${ }^{12}$

Previous theoretical investigations of disilenes have concentrated on the geometry and stability of disilene, $\mathrm{Si}_{2} \mathrm{H}_{4}$, in the singlet state, relative to its geometry and stability in the lowest lying triplet state. ${ }^{13-32}$ Michl and Raabe, in their 1985 review of silene chemistry, pointed out that the stability of singlet disilene relative to the triplet state has been underestimated in the early theoretical work ${ }^{1 e}$ whereas Teramae ${ }^{26}$ concluded that the ground-state electronic structure of disilene involves a significant contribution from the diradical (or triplet) state. Teramae also calculated longer Si-Si bond distances and larger deviations from planarity than those previously reported. ${ }^{26}$ Few calculations have been done on substituted disilenes. ${ }^{17 b}, 19,29,32$ KroghJespersen investigated tetramethyldisilene, $\mathrm{Si}_{2}\left(\mathrm{CH}_{3}\right)_{4}$, and found that the ground state is a singlet with a trans-bent geometry. ${ }^{17 \mathrm{~b}}$ The state corresponding to the planar geometry is $1.0 \mathrm{kcal} / \mathrm{mole}$ higher in energy. Inclusion of $d$-orbital functions in the silicon basis set reverses these states.

The goal of this study is to investigate the bonding in the known disilenes and to compare

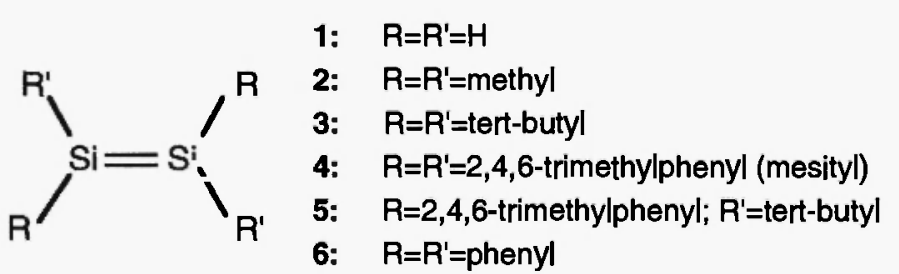

their electronic structures with those of the corresponding alkenes in order to explain the experimentally observed similarities (reactivity patterns) and differences (electronic spectra and 
rotational barriers). Calculations were performed on three known disilenes: tetra-( $t$-butyl)disilene, 3, tetramesityldisilene, 4, and trans-1,2-di-(t-butyl)-1,2-dimesityldisilene, 5. Calculations were also performed on disilene, $\mathrm{S}_{2} \mathrm{H}_{4}, 1$, and tetramethyldisilene, 2 , in order to detect any trends upon substitution of bulkier substituents, as well as on tetraphenyldisilene, 6 , to determine the effect of an unsubstituted aryl group. For each disilene compound the calculations were done twice: with the minimal basis set and with an extended basis set that included d-orbital functions on Si.

\section{METHODS}

Molecular orbital calculations were carried out on a VAX 8600 computer system using the Fenske-Hall molecular orbital model. ${ }^{33}$ This method is based on a nonparameterized, nonempirical approximate Hartree-Fock-Roothaan procedure. The results of each calculation (eigenvalues and eigenvectors) are completely determined by the geometry of the molecule and the nature and size of the basis set.

Basis Functions: Clementi's free atom double- $\zeta$ Hartree-Fock functions ${ }^{34}$ were used for carbon and silicon. All but the valence shell p-functions were curve-fit to single- $\zeta$ form by use of the criterion of maximum overlap. ${ }^{35}$ A value of 1.16 was used for the hydrogen atom $1 \mathrm{~s}$ orbital exponent. $^{36}$ The exponent for the Si d-orbitals was set to 1.38 .

Geometry: The purpose of this study is to chart the effect of different substituents on the Si-Si interaction. This is done primarily by examining the total orbital overlap population and the $\pi$ orbital overlap population between the Si atoms. In order for any comparison to be valid the changes in overlap population must not be due to differences in Si-Si bond distances, therefore in all calculations the Si-Si bond distance was held constant at $2.143 \AA$ so that comparisons of overlap populations would be valid. Other molecular geometries (C-Si bond lengths and C-Si-C bond angles) for 4 and 5 were taken from $\mathrm{x}$-ray crystal structure data.2c, 8 The geometry (C-Si bond lengths and $\mathrm{C}-\mathrm{Si}-\mathrm{C}$ bond angles) of 3 was constructed from the data for 5 . The geometry (C$\mathrm{Si}$ bond lengths and $\mathrm{C}-\mathrm{Si}-\mathrm{C}$ bond angles) of 2 was constructed using the same $\mathrm{C}-\mathrm{Si}-\mathrm{C}$ bond angle that was used for 3 . In addition, a Si-C bond length of $1.904 \AA$, a C-H bond length of $1.09 \AA$, and an $\mathrm{H}-\mathrm{C}-\mathrm{H}$ bond angle of $105^{\circ}$ was used. The optimum planar geometry ( $\mathrm{Si}-\mathrm{H}$ bond lengths and $\mathrm{H}-$ $\mathrm{Si}-\mathrm{H}$ bond angles) of 1 as determined by Krogh-Jespersen in 1985 was used. ${ }^{17 \mathrm{~b}}$

Fragment Orbital Analysis: All calculations except those for $\mathrm{C}_{2} \mathrm{H}_{4}$ and 1 were performed using the transformed basis approach. ${ }^{33 d, 37}$ A molecular orbital analysis was performed on each substituent, treated as a neutral radical (e.g., Me•, $t$-butyl•, and mes०). The resulting fragment molecular orbitals were used as a basis set for the calculations on the full molecule. This approach greatly simplifies the interpretation of the results for systems with large substituents.

\section{RESULTS AND DISCUSSION}

Minimal Basis Set. The results of the calculations on 1 and 2 indicate that the bonding in these molecules can best be described as being analogous to the bonding in the corresponding alkenes. There are no substantial differences between the $\mathrm{C}$ - and Si- multiply bonded species. The most important result, for the purposes of this study, is that the HOMO in each case is a pure Si $p \pi$-p $\pi$ bonding molecular orbital and that the LUMO is the corresponding antibonding molecular orbital. The HOMO is stabilized by approximately $3 \mathrm{eV}$ relative to the Si p-orbitals (approximately $2 \mathrm{eV}$ in the case of the methyl substituted disilene) and there is no evidence to suggest that a diradical structure would be favored at this level of approximation.

Two competing effects are observed as the size of the alkyl substituent is increased from $\mathrm{H}$ (in 1) to $\mathrm{CH}_{3}$ (in 2) to $\mathrm{C}\left(\mathrm{CH}_{3}\right)_{3}$ (in 3). The first effect is that the energy of the HOMO (in all cases a Si-Si $\pi$-bonding orbital) increases relative to the energy of the Si atomic p-orbitals (Table I). The $\pi$ bond is less stabilized (relative to the atomic p orbitals) in the bulkier $\mathbf{3}$ than in $\mathbf{1}$ or 2 . The second effect is that the total overlap population ${ }^{38}$ between the Si atoms increases, implying that the alkyl groups are acting as electron donating groups (Table II). There is also significant interaction between the Si 3 s orbitals and the lower energy $t$-butyl fragment orbitals, thus further stabilizing the molecule.

In tetramesityl disilene, $\mathbf{4}$, the picture is slightly altered. The total overlap population between the Si atoms is substantially lower than that observed in $\mathbf{2}$ and $\mathbf{3}$, indicating that the 
phenyl rings are acting as electron-withdrawing groups. There are two important stabilizing effects, however, that are related to the molecular geometry. The solid-state geometry of 4 is such that two of the mesityl groups cis to each other are approximately in the same plane as the $\mathrm{C}_{4} \mathrm{Si}_{2}$ core, whereas the other two mesityl rings are approximately perpendicular to this plane (Figure

\begin{tabular}{|c|c|c|c|c|c|c|}
\hline \multicolumn{7}{|c|}{$\begin{array}{c}\text { Table l. Calculated Energy Differences and Observed Electronic } \\
\text { Transitions in Several Disilenes }\end{array}$} \\
\hline \multirow{3}{*}{$\frac{\text { Molecule }}{1}$} & \multicolumn{2}{|c|}{ Stabilization Energy ${ }^{b}$} & \multicolumn{2}{|c|}{ HOMO-LUMO Gap } & \multirow{2}{*}{\multicolumn{2}{|c|}{$\frac{\text { Obsenved Transitions }}{\lambda \max (n m) \Delta E_{\text {obs }}(e V)}$}} \\
\hline & $\begin{array}{l}\text { without } \\
\text { d-orbitals }\end{array}$ & $\begin{array}{l}\text { with } \\
\text { d-orbitals }\end{array}$ & $\begin{array}{l}\text { without } \\
\text { d-orbitals }\end{array}$ & $\begin{array}{l}\text { with } \\
\text { d-orbitals }\end{array}$ & & \\
\hline & 3.06 & 3.15 & 9.18 & 4.36 & & \\
\hline 2 & 1.86 & 2.26 & 10.68 & 3.80 & $345^{c}$ & 3.59 \\
\hline 3 & 1.06 & 1.68 & 11.28 & 3.92 & $433^{d}$ & 2.86 \\
\hline 4 & 0.34 & 1.09 & 7.94 & 3.23 & $420^{C}$ & 2.95 \\
\hline 5 & 0.76 & 1.49 & 10.92 & 3.56 & $332^{e}$ & 3.69 \\
\hline \multicolumn{7}{|c|}{ 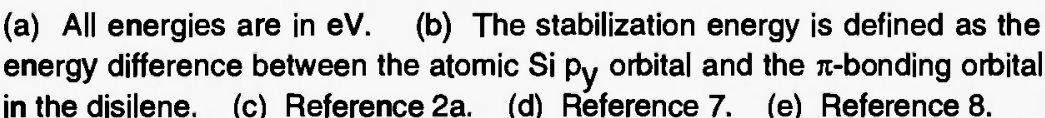 } \\
\hline
\end{tabular}

1a). While this is an adequate steric arrangement, one can imagine other less sterically hindered geometries. 39 The HOMO in 4 is a conjugated $\pi$ system comprising both $\mathrm{Si}$ atoms and the $\pi$ orbitals of the two mesityl rings that are approximately coplanar with the $\mathrm{S}_{2} \mathrm{C}_{4}$ framework. This orbital is both Si-Si bonding and Si-ring bonding. The LUMO is Si-Si antibonding and Si-ring bonding. The phenyl-Si-Si-phenyl conjugation apparently weakens the Si-Si bond, as evidenced by the decreased Si-Si overlap population, but provides some stabilization of the molecule due to delocalization.

The ortho-methyl groups on the mesityl rings that are oriented periperpendicularly to the molecular plane contribute to the stability of the molecule. There is a substantial agostic interactions between all four of these methyl groups and the SiSi bond (Figure 1) which provides some electron-donating alkyl functionality to offset the electron withdrawing aryl groups. This observation may help to explain why disilenes with 2,6disubstituted aryl groups as substituents have been isolated but tetraphenyl disilene itself has not been isolated.

In order to further study the effect of aromatic ring substituents, calculations were done on tetraphenyl disilene in two geometries, one corresponding to the mesityl geometry (two rings approximately planar and two rings approximately perpendicular to the Si-Si bond), 6a, and one corresponding to all rings rotated approximately $75^{\circ}$ relative to the plane, $6 \mathrm{~b}$. The total overlap population between the two Si atoms decreases substantially in both $6 \mathrm{a}$ and $\mathbf{6 b}$ relative to the total overlap population in 4 (Table II). This provides strong evidence in support of the stabilizing effect of alkyl substituents in the orthopositions of the aromatic ring.

In the mixed substituted disilene, 5, the mesityl groups are oriented perpendicularly to the Si-Si $\pi$ bond in the solid state (Figure 1b) and do not contribute to any $\pi$ delocalization. The effect of this is seen dramatically in the large increase in the SiSi overlap population as compared with that of 4. The HOMO in this system is a pure $\mathrm{Si}-\mathrm{Si} \pi$-bond, analogous to what was observed in the tetra-alkyl substituted systems, but there is still a strong interaction between the ortho-methyl groups on the two

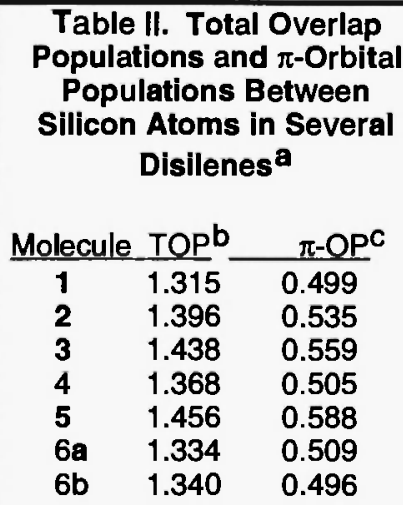

(a) The Si-Si bond distance in each molecule is the same: $2.143 A$, to allow for comparison of overlap populations. (b) See reference 38 . (c) $\pi$-orbital population is the overlap population between the Iwo silicon atoms in the $\pi$ bonding orbital only. remaining mesityl rings and the $\mathrm{Si}$ atoms.

Expanded Basis Set. The composition and relative ordering of the occupied molecular orbitals are not substantially altered when $d$-orbitals are included in the Si basis set relative to what is observed in the minimal basis set calculations. A minor mixing of Si d-orbital character in the $\pi$ - 


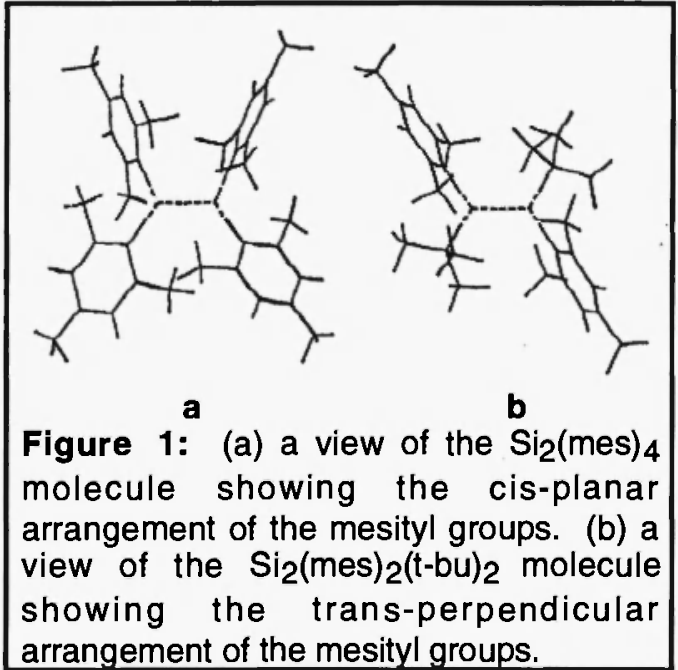

bonding HOMOs occurs in several of the molecules studied. This mixing lowers the energies of the HOMOs slightly more than what is observed in the other occupied molecular orbitals.

The compositions and energies of the virtual (unoccupied) molecular orbitals are significantly altered when Si d-orbitals are included in the basis set. 41 The substantial addition of Si d-orbital character in the LUMO significantly lowers its energy such that a large decrease in the HOMO-LUMO gap occurs in each molecule (Table I) along with a marked change in the nature of the LUMO. The effect of altering the character of frontier orbitals in main-group compounds by including d-orbital functions in the basis set of the main-group element is not unprecedented. Similar results have been obtained in molecules as diverse as $\mathrm{SO}_{2}, \mathrm{H}_{2} \mathrm{~S}, 42$ $\mathrm{CH}_{2} \mathrm{Cl}_{2}$, and $\mathrm{CH}_{3} \mathrm{l}^{43}$

The decreased HOMO-LUMO gap correlates much better with the experimentally observed electronic transitions of the known disilenes that occur in the visible region of the spectrum (Table I). 44 Using the minimal basis set the HOMO-

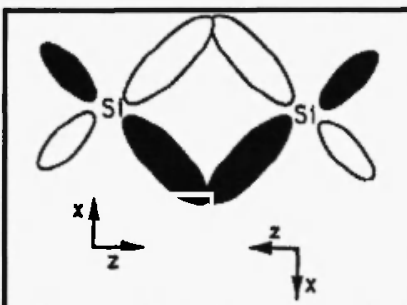

Figure 2: The $\pi_{x}$ orbital, a mixture of Si $3 d_{x z}$ and $3 p_{x}$ orbital character, which is the LUMO of all the disilene molecules when the expanded basis set of $\mathrm{Si}$ is used in the calculations. LUMO gap for the disilenes is of the same magnitude as that observed for alkenes. Alkenes generally exhibit $\pi-\pi^{*}$ transitions in the ultraviolet region of the spectrum.

Inclusion of the d-orbitals on $\mathrm{Si}$ also changes the composition of the LUMO from a Si $p_{y}$ - Si $p_{y} \pi^{*}$-antibonding orbital to a $\pi$ bonding orbital that is orthogonal to the HOMO and composed of a mixture of Si $p_{x}$ and $S i d_{x z}$ orbital character (Figure 2). The mixing of the d-orbital character with the $p_{x}$ orbital allows the $\pi$ bond to be localized between the two silicon atoms and directed away from the substituents.

The lower rotation barrier of disilenes compared to that of alkenes may be attributed to both the smaller HOMO-LUMO gap and the altered character of the LUMO. We have not performed a population analysis in the excited state yet so these results cannot be compared with the results of the more accurate ab-initio methods for $\mathrm{Si}_{2} \mathrm{H}_{4}$. However, it is possible that because of the smaller HOMO-LUMO gap a significant number of molecules may be in the first electronic excited state, $\left(\pi_{y}\right)^{1}\left(\pi_{x}\right)^{1}$, rather than the

ground state, $\left(\pi_{\mathrm{y}}\right)^{2}$, at room temperature. In the first excited state the $\pi$-bond in the molecule is essentially two half $\pi$-bonds with one of the half $\pi$-bonds having significantly higher energy. This will lead to an overall weaker $\pi$-bond than what would occur in the ground state, thereby lowering the rotation barrier.

Summary. Our calculations show that the bonding in disilenes is very sensitive to the substituents on the silicon atoms. Bulky substituents on the isolated disilenes serve not only to protect the SiSi double bond sterically but also to stabilize the bond electronically. Alkyl groups are more stabilizing than aryl groups, providing greater electron donation into the double bond. Aryl substituents appear to be electon withdrawing, and therefore destablizing. The enhanced reactivity of disilenes compared with that of alkenes may be attributed to the existence of low-lying unoccupied orbitals that have substantial Si d-orbital character. These virtual orbitals may also provide a low-energy pathway for rotation about the Si-Si axis. 
Acknowledgments: We thank Professor R.F. Fenske for helpful discussions. This research was initiated while H.A.H. was a visiting faculty member at Grinnell College, Grinnell, IA, and was supported in part by a grant from Research Corporation. H.A.H. gratefully acknowledges the support of the Clare Boothe Luce Fund, administered by the Henry Luce Foundation

\section{REFERENCES}

(1) For recent reviews see: (a) Raabe, G.; Michl, J. in The Chemistry of Organic Silicon Compounds (S. Patai and Z. Rappaport, Eds.) Vol. 2, Chap. 17,John Wiley and Sons, Ltd 1989, p.1015. (b) Apeloig, Y. op cit, Vol. 1, Chap. 2, p. 57. (c) West, R. Angew. Chem. Int. Ed., Eng. 1987, 26, 1201-1211. (d) Cowley, A.C.; Norman, N.C. Prog. Inorg. Chem. 1986, 34, 1-63. (e) Raabe, G.; Michl, J. Chem. Rev. 1985, 85, 419-509.

(2) (a) West, R.; Fink, M.J.; Michl, J. Science, 1981, 214, 1343. (b) Fink, M.J.; Michalczyk, M.J.; Haller, K.J.; West, R.; Michl, J. J. Chem. Soc., Chem. Commun. 1983, 1010. (c) Fink, M.J.; Michalczyk, M.J.; Haller, K.J.; West, R.Organometallics, 1984, 3, 793.

(3) (a) Masamune, S.; Hanzawa, Y.; Murakami, S.; Bally, T.; Blount, J.F. J. Am. Chem. Soc. $1982,104,1150$.

(4) Masamune, S.; Murakami, S.; Snow, J.T.; Tobita, H.; Williams, D.J. Organometallics, 1984, 3, 333.

(5) Watanabe, H.; Okawa, T.; Kato, M.; Nagai, Y. J. Chem. Soc., Chem. Commun. 1983, 781.

(6) Matsumoto, H.; Arai, T.; Watanabe, H.; Nagai, Y. J. Chem. Soc., Chem. Commun. 1984, 724.

(7) (a) Masamune, S.; Tobita, H.; Murakami, S. J. Am. Chem. Soc. 1983, 105, 6524. (b) Masamune, S.; Murakami, S.; Tobita, H. Organometallics, 1983, 2, 1464.

(8) Michalczyk, M.J.; West, R.; Michl, J. J. Am. Chem. Soc. 1984, 106, 821.

(9) Watanabe, H.; Takeuchi, K.; Fukawa, N.; Kato, M.; Goto, M.; Nagal, Y. Chem. Lett. 1987, 7, 1341.

(10) Shephard, B.D.; Powell, D.R.; West, R. Organometallics, 1989, 8, 2664.

(11) There are two structurally characterized disilenes shown to be completely planar: trans- 1,2dimesityl-1,2-di(t-butyl)disilene ${ }^{8}$ and trans-1,2,-dimesityl- 1,2-diadamantyldisilene ${ }^{12}$.

(12) See for example: Cotton, F.A.; Wilkinson, G.; Gaus, P.L. Basic Inorganic Chemistry, 2nd Ed.; John Wiley and Sons: New York, 1987 (p. 332).

(13) Snyder, L.C.; Wasserman, Z.R. J. Am. Chem. Soc. 1979, 101, 5222.

(14) Poirier, R.A.; Goddard, J.D. Chem. Phys. Lett. 1981, 80, 37.

(15) Lischka, H.; Kohler, H.-J. Chem. Phys. Lett. 1982, 85, 467.

(16) Kohler, H.-J.; Lischka, H. J. Am. Chem. Soc. 1982, $104,5884$.

(17) (a) Krogh-Jespersen, K. J. Phys. Chem. 1982, 86, 1492. (b) Krogh-Jespersen, K. J. Am. Chem. Soc. 1985, 107, 537.

(18) (a) Nagase, S.; Kudo, T. J. Mol. Struct. (THEOCHEM), 1983, 103, 35. (b) Organometallics, $1984,3,1320$.

(19) Schoeller, W.W.; Staimmler, V. Inorg. Chem. 1984, 23, 3369.

(20) Ohta, K.; Davidson, E.R.; Morokuma, K. J. Am. Chem. Soc. 1985, 107, 3466.

(21) Gordon, M.S.; Truong, T.N.; Bonderson, E.K. J. Am. Chem. Soc. 1986, 108, 1421.

(22) Luke, B.T.; Pople, J.A.; Krogh-Jespersen, M.-B.; Apeloig, Y.; Karni, M.; Chandrasekhar, J.; Schleyer, P.v.R. J. Am. Chem. Soc. 1986, 108, 270.

(23) Ho, P.; Coltrin, M.E.; Binkley, J.S.; Melius, C.F. J. Phys. Chem. 1986, 90, 3399.

(24) Tossel, J.A.; Lazzeretti, P. Chem. Phys. Lett. 1986, 128, 420.

(25) Olbrich, G. Chem. Phys. Lett. 1986, 130, 115.

(26) Teramae, H. J. Am. Chem. Soc. 1987, 109, 4140.

(27) (a) Schmidt, M.W.; Truong, P.N.; Gordon, M.J. J. Amer. Chem. Soc., 1987, 109, 5217. (b) Hrovat, D.A.; Sun, H.; Borden, W.T. J. Mol. Struct. (THOECHEM), 1988, $163,51$.

(28) Trinquier, G.; Malrieu, J.P. J. Amer. Chem. Soc. 1987, 109, 5303.

(29) (a) Malrieu, J.P.; Trinquier, G. J. Amer. Chem. Soc., 1989, 111, 5916. (b) Trinquier, G. J. Phys. Chem., 1990, 94, 6184.

(30) Liang, C.; Allen, L.C. J. Amer. Chem. Soc., 1990, 112, 1039.

(31) (a) Trinquier, G. J. Amer. Chem. Soc., 1990, 112, 2150. (b) Trinquier, G.; Malrieu, J.P. J. Amer. Chem. Soc., 1991, 113, 8634.

(32) Karni, M.; Apeloig, Y. J. Amer. Chem. Soc. 1990, 112, 8589. 
(33) (a) Hall, M.B.; Fenske, R.F. Inorg. Chem. 1972, 11, 768. (b) Fenske, R.F. Prog. Inorg. Chem. 1976, 21, 179. (c) Fenske, R.F. Pure Appl. Chem. 1971, 27, 61. (d) Fenske, R.F. Pure \& Appl. Chem. 1988, 60, 1153.

(34) Clementi, E.; Ramondi, D.L. J. Chem. Phys. 1963, 38, 2686-2689.

(35) Radtke, D.D. Ph.D. Dissertation, University of Wisconsin-Madison, 1966.

(36) Hehre, W.J.; Stewart, R.F.; Pople, J.A. J. Chem. Phys. 1969, 51, 2657-2664.

(37) Hoffmann, R. Science, 1981, 211, 995 and references cited therein.

(38) TOP $=\sum_{i} \sum_{n_{k} ?} \sum_{s_{1}} 2 N(i) C_{n_{k}} C_{s l} S\left(r_{k}, s_{1}\right)$ where $C_{k_{k}}$ and $C_{s_{1}}$ are coefficients of valence orbitals on atoms $k$ and $I$ in the ith $M O$, populated with $N(i)$ electrons. $S\left(r_{k}, s_{l}\right)$ denotes orbital overlap; and the first summation is over all occupied MOs. It is noteworthy that TOP is a measure of the degree of covalency (i.e., bonding electron density) between atoms.

(39) West and coworkers have recently published another X-ray crystal structure of 4.40 The ring orientations in the unsolvated 4 are similar to those in the solvated $4.3 \mathrm{c}$ The major difference between the two structures appears to be that the Si atoms are less pyramidalized and the Si-Si bond is less twisted in the most recent, unsolvated, structure.

(40) Shephard, B.D.; Campana, C.F.; West, R. Heteroatom Chemistry, 1990, 1, 1.

(41) The role of d-orbitals (as opposed to d-functions) in bonding of main-group elements is quite controversial ${ }^{42}$ however the type of effect observed in this study is not unprecedented. 43

(42) Magnusson, E. J. Amer. Chem. Soc. 1990, 112, 7940.

(43) Czech, P.T.; Gladysz, J.A.; Fenske, R.F. Organometallics 1989, 8, 1806.

(44) The excitation energy is not simply the difference in energy between the HOMO and the LUMO, but is given by the following:45 $\Delta \mathrm{E}=\left(\varepsilon_{1}-\varepsilon_{2}\right)-\left(\mathrm{J}_{12}-\mathrm{K}_{12}\right) \pm \mathrm{K}_{12}$. Therefore, the HOMO-LUMO separation should overestimate the excitation energy by an amount equivalent to $J_{12}$, the coulomb integral, on average. Even taking this factor into account, inclusion of $d$ orbitals on Si still provides better agreement with experimental data.

(45) Roothaan, C.C.J. Rev. Mod. Phys. 1951, 23, 69.

Received: August 8, 1993 - Accepted: August 25, 1993 - Accepted in revised camera-ready format: September 14, 1993 